\section{Alternative Explanations of the Eötvös Results}

Our recent reanalysis ${ }^{1,2}$ of the experiment by Eötvös, Pekár, and Fekete ${ }^{3}$ (EPF) has uncovered in the EPF data a correlation between the fractional acceleration difference $\Delta \kappa$ and the quantity $\Delta(B / \mu)$, where $B$ is baryon number and $\mu$ is the mass in units of $m\left({ }_{1} \mathrm{H}\right)$. Although this correlation agrees with what one would expect from the presence of an intermediate-range force whose source is baryon number of hypercharge, the possibility remains that the EPF results could be explained in terms of conventional physics. The only alternative model we know of at present which has a serious chance of explaining these results is the "thermal-gradient" model of Chu and Dicke $^{4}(C D)$, and for this reason the CD model deserves to be taken seriously. In what follows we examine the strengths and limitations of the $C D$ model in light of the EPF data.

The premise of this model is that if horizontal thermal gradients were present in the EPF apparatus, they could produce a gentle "breeze" which could exert a force on the samples being compared. Since the samples, or the containers they were in, had different physical dimensions, the forces exerted on opposite sides of the apparatus would not be equal, and a net torque could result. In practice, this would lead to a correlation between $\Delta \kappa$ and $\Delta(1 / \rho)$ or $\Delta S$, where $\rho$ is the density of the sample and $S$ is the cross-sectional surface area of the sample or its container. This nicely illustrates how a systematic effect can appear to depend on a property of the samples, such as $1 / \rho$.

It is useful to picture any acceptable alternative to the baryon-number or hypercharge hypothesis as satisfying two separate criteria: (i) To start with, the mechanism in question should have the property that the torque which it produces must change when the EPF apparatus is rotated through $180^{\circ}$; (ii) the torque produced by the alternative mechanism must not only be composition dependent, but also must vary from one material to another in a manner that (at least approximately) simulates a variation with $B / \mu$. The significance of the first criterion, when applied to the $C D$ model, is to suggest that if a thermal gradient (or breeze) is to simulate the effects of a matter distribution (e.g., the presence of a mountain or building), it must produce a force which is similarly constant on average temporally relative to the apparatus. However, it is unclear how any likely heat source (e.g., a window or radiator) would always produce a gradient with both a fixed direction and fixed magnitude, independent of time of day or year over the period of months or years that the experiment took place. The challenge to the CD model arising from the second criterion is to explain adequately the $\mathrm{Pt}$ data, which tend not to fit that well, irrespective of how the model is formulated. As CD correctly point out, there is a clear suggestion of a correlation between $\Delta \kappa$ and $\Delta S$ or $\Delta(1 / \rho)$ evidenced by the double-torsion-balance data alone, particularly for those comparisons carried out using EPF's method III, which happened not to involve Pt. The reason for this is illuminating, and provides an insight into other possible correlations as well. If we examine a plot of $B / \mu$ as a function of atomic number $Z$, we see that for the EPF samples $B / \mu$ is an approximately monotonically increasing function of $Z$, provided we exclude Pt. It follows that since $B / \mu$ does in fact correlate with $\kappa$, so will any other nearly monotonically changing variable, provided that the $\mathrm{Pt}$ data are excluded. Hence it is precisely these data which test the characteristic shape of $B / \mu$ as a function of $Z$, and which thus discriminate between a correlation with $B / \mu$ and one involving some other variable. It follows that the suggestion of an approximate correlation between $\Delta \kappa$ and $\Delta(1 / \rho)$ is not surprising, since $-1 / \rho$ is also an approximately increasing function of $Z$ for the substances studied by EPF.

In summary, the $C D$ model is very clever and sufficiently promising to warrant more detailed study, should ongoing experiments fail to confirm the original EPF results. The issues that it must address more fully are mechanisms for producing a temporally constant thermal gradient over a long period of time, and the behavior of the Pt data.

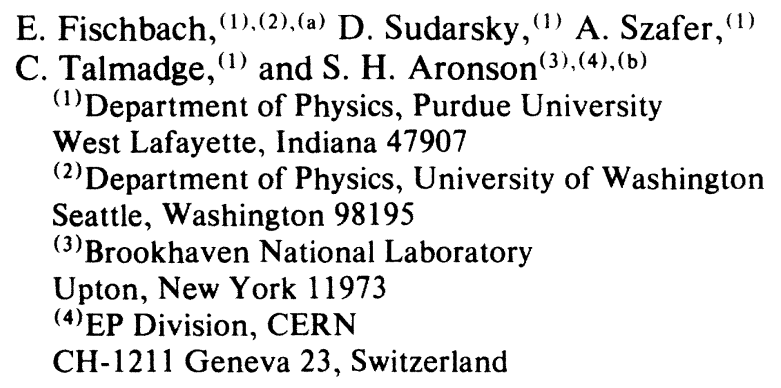

Received 7 July 1986

PACS numbers: 04.90 . te

(a) Present address: Department of Physics, Purdue University, West Lafayette, IN 47907.

(b) Present address: Brookhaven National Laboratory, Upton, NY 11973.

${ }^{1}$ E. Fischbach, D. Sudarsky, A. Szafer, C. Talmadge, and S. H. Aronson, Phys. Rev. Lett. 56, 3 (1986).

${ }^{2}$ E. Fischbach, D. Sudarsky, A. Szafer, C. Talmadge, and S. H. Aronson, in Proceedings of the Second Conference on Interactions Between Particle and Nuclear Physics-1986, edited by D. Geesaman, AIP Conference Proceedings No. 150 (American Institute of Physics, New York, 1986), p. 1102.

${ }^{3}$ R. v. Eötvös, D. Pekár, and E. Fekete, Ann. Phys. (Leipzig) 68, 11 (1922), and in Roland Eötvös Gesammelte Arbeiten, edited by P. Selényi (Akadémiai Kiado, Budapest, 1953), pp. 307-372.

${ }^{4}$ S. Y. Chu and R. H. Dicke, this issue [Phys. Rev. Lett. $57,1823(1986)]$. 